\title{
Atazanavir and Cardiovascular Risk Among Human Immunodeficiency Virus-Infected Patients: A Systematic Review
}

\author{
Dominic Chow · Cecilia Shikuma · Corey Ritchings · Muxing Guo • \\ Lisa Rosenblatt
}

Received: June 27, 2016 / Published online: September 27, 2016

(c) The Author(s) 2016. This article is published with open access at Springerlink.com

\begin{abstract}
Introduction: Patients with human immunodeficiency virus (HIV) infection have an increased risk of cardiovascular disease (CVD). While viral suppression with antiretroviral therapy decreases CVD risk overall, several studies have suggested that certain antiretrovirals, particularly certain protease inhibitors, may be associated with an increased relative risk of CVD. In AIDS Clinical Trials Group $5260 \mathrm{~s}$, ritonavir-boosted atazanavir (ATV) was associated with slower atherosclerosis progression compared to ritonavir-boosted darunavir and raltegravir,
\end{abstract}

Enhanced content To view enhanced content for this article go to http://www.medengine.com/Redeem/ E0E6F0607F342B32.

Electronic supplementary material The online version of this article (doi:10.1007/s40121-016-0132-z) contains supplementary material, which is available to authorized users.

D. Chow $(\bowtie) \cdot C$. Shikuma

Hawaii Center for AIDS, John A. Burns School of

Medicine, University of Hawaii Mānoa, Honolulu,

HI, USA

e-mail: dominicc@hawaii.edu

C. Ritchings · M. Guo · L. Rosenblatt

Bristol-Myers Squibb, Plainsboro, NJ, USA potentially due to hyperbilirubinemia. Although hyperbilirubinemia may lead to increased rates of treatment discontinuation, it may also contribute to a favorable cardiovascular (CV) profile for ATV. To fully elucidate the effect of ATV on CVD risk among HIV-infected patients, a systematic review of the literature was performed.

Methods: A systematic search of the PubMed and Embase databases was conducted on August 26, 2015, using terms to identify papers that discuss ATV, HIV, and CVD. Articles were limited to English-language publications of randomized-controlled or observational studies investigating adult humans. The primary outcome was the incidence of CVD. Articles describing surrogate markers of CVD were also included.

Results: Ten studies were included in this qualitative analysis: six reported CVD outcomes, two reported data on atherosclerosis as assessed by carotid intima-media thickness (cIMT), and two reported outcomes related to endothelial function. The studies reporting the incidence of myocardial infarction (MI) among HIV-infected patients showed that ATV (boosted and unboosted) was not associated 
with an increased risk of acute MI. Other CV endpoints were similarly unaffected by treatment with ATV. Compared with non-ATV-based regimens, ATV had beneficial effects on cIMT progression in the publications identified, with no apparent impact on endothelial function.

Conclusions: This analysis showed that there was no increased risk or occurrence of adverse CV events among HIV-infected patients receiving ATV. Markers of atherosclerosis were improved, suggesting a possible antioxidant effect of ATV, and endothelial function was not affected.

Funding: Bristol-Myers Squibb (article processing charges and medical writing support).

Keywords: Antiretroviral therapy; Atazanavir; Atherosclerosis; Cardiovascular disease; Carotid intima-media thickness; cIMT; Endothelial function; Flow-mediated dilation; HIV

\section{INTRODUCTION}

Advances in antiretroviral therapy (ART) for the treatment of human immunodeficiency virus (HIV) have meant that patients can achieve a life expectancy near to normal [1]. However, HIV-infected patients are known to have an increased risk of cardiovascular disease (CVD) compared with the general population, with a significantly elevated mortality rate from cardiovascular (CV) events [2].

The cause of the increased CVD risk in HIV-infected patients is multifactorial, involving traditional CVD risk factors, HIV infection-associated risk factors, and ARTassociated risk factors [3-6]. With improved life expectancy, increased exposure to traditional CVD risk factors, such as tobacco use, dyslipidemia, hypertension, and diabetes, occurs [3]. HIV infection is associated with increased inflammation, immune dysfunction, and impaired fibrinolysis [5]. While viral suppression with ART has been associated with an overall reduced risk of CVD events compared to untreated HIV [7], several studies have suggested that certain antiretrovirals (ARs) are associated with an increased relative risk of CVD [3, 8-10], possibly linked to factors, such as the development of insulin resistance, dyslipidemia, and altered fat distribution [6]. Abacavir (ABC), didanosine, indinavir, and ritonavir-boosted lopinavir (LPV/r) have been shown to be associated with an increased risk of myocardial infarction (MI) $[8,10]$. Some studies suggest an association between protease inhibitors (PIs) as a drug class and increased CV events among HIV-infected patients, which may be attributable to the proatherogenic effect of these agents on lipids $[3,9]$. Importantly, these early studies did not differentiate between different types of PIs. Other analyses investigating CV risk and PIs have been mixed, with observational trials, but not randomized studies, indicating a relationship between some PIs and increased risk of CVD [8].

In contrast, the PI atazanavir (ATV) is associated with improved lipid profiles, both in patients with HIV and those with type 1 diabetes without HIV, and improved endothelial function in patients with type 2 diabetes without HIV [11-13]. Ritonavir-boosted ATV $(\mathrm{ATV} / \mathrm{r})$ has also been associated with slower progression of carotid intima-media thickness (cIMT)—a surrogate marker for CVD riskcompared with ritonavir-boosted darunavir (DRV/r) and raltegravir (RAL) [14], potentially due to increased bilirubin caused by ATV. Bilirubin is a known antioxidant that has anti-atherosclerotic properties [15]. At the same time, ATV-associated hyperbilirubinemia 
may lead to increased treatment discontinuations, as was shown in AIDS Clinical Trials Group (ACTG) A5257, where $\mathrm{ATV} / \mathrm{r}$ was equivalent to $\mathrm{DRV} / \mathrm{r}$ and RAL in risk for virologic failure, but was associated with more discontinuations due to adverse events, leading to the overall inferiority [16].

To fully elucidate the effect of ATV on CVD risk among HIV-infected patients so as to enable a more complete assessment of its risk/benefit profile, a systematic review of the literature was performed. The aim of this qualitative analysis was to assess CV outcomes among patients with HIV treated with ATV, using both CV event and surrogate marker data, where available. Surrogate markers of atherosclerosis and endothelial dysfunction were included, as both have shown to be independent predictors of CV events [17-20].

\section{MATERIALS AND METHODS}

\section{Search Criteria}

The PubMed and Embase databases were used for the literature search, which was conducted on August 26, 2015. The PubMed search was performed using free text fields and Medical Subject Heading terms to capture studies in patients with HIV infection receiving ART who had experienced adverse $\mathrm{CV}$ events (such as coronary artery disease, MI, ischemia, angina, cerebrovascular accident, transient ischemic attack, or peripheral vascular disease). A similar search was conducted using Embase. For detailed terms used, see the Supplementary Material.

For both searches, citations were limited to English-language articles with a publication type of clinical trial, meta-analysis, observational study, cohort study, or case-control study investigating adult humans; no date restriction was placed on the searches. The reference lists of all included papers were hand-searched to identify additional papers. While systematic reviews and meta-analyses were not included in the qualitative analysis, the reference lists of these articles were hand-searched to ensure all eligible studies were identified.

\section{Study Selection}

Study selection occurred in a two-step process: (1) eligibility was initially assessed using titles and abstracts and (2) those that were not excluded were screened for inclusion/exclusion using the full text. Articles excluded from the analysis were retrospective studies, narrative reviews, editorials, pharmacokinetic studies, pilot studies, animal or in vitro studies, studies in children, and studies in which only the change in lipid profile was reported. Studies of patient subpopulations were excluded unless the population characteristics were generalizable to the adult HIV population. Studies without an ATV arm and those not reporting on outcomes of interest were excluded.

The primary outcome was the incidence of CVD or CV event, defined as coronary heart or artery disease, MI, acute coronary syndrome, thromboembolism, stroke or cerebrovascular events, atherosclerosis, and coronary procedures, such as percutaneous coronary intervention and coronary artery bypass graft. Articles describing surrogate markers of atherosclerosis and endothelial function were also included.

\section{Data Extraction and Assessment of Study Quality}

Data collected from the articles included study description and trial characteristics, patient characteristics, treatment characteristics, and results/endpoints. Data extraction and 
validation were performed by MG and LR, respectively. The results of the data extraction were independently reviewed by DC and CS prior to commencing writing of the article. The quality of the included studies was assessed using the methodology checklist of the 2012 National Institute for Health and Care Excellence Guidelines Manual [21] for the randomized-controlled trials and the 'checklist for measuring study quality' [22] for the cohort studies.

\section{Compliance with Ethics Guidelines}

This article is based on previously conducted studies and does not involve any new studies of human or animal subjects performed by any of the authors.

\section{RESULTS}

A preferred reporting items for systematic reviews and meta-analyses flow diagram showing the screening process and reasons for exclusion is presented in Fig. 1. A total of 24 studies were identified using PubMed, 77 were identified using Embase, and five were identified from hand searching the reference lists of identified articles. The major reason for excluding articles during the first screening round was that they were not a clinical or cohort study; during the second round of screening, not reporting an outcome of interest was the main reason for article exclusion. In total, ten studies met all criteria and were included in the qualitative analysis (Fig. 1). Among the included studies, the length of follow-up ranged from 1.5 to 4.8 years, and the studies varied greatly with respect to the ATV-containing and comparator regimens used (Tables 1, 2, 3).
Quality assessment of the included studies is shown in detail in the Supplementary Material. The included randomized-controlled trials were of moderate quality, mainly because they were either fully open-label studies [14, 23-25] or were only partially blinded to one component of the AR regimen [26] or to observers [27]. In addition, one trial showed a significant elevation in triglycerides at baseline in the ATV arm [27] and two trials showed an imbalance in discontinuation rates between the ATV and comparator arms [14, 24]. The included cohort studies were of moderate quality, mainly due to the lack of information on patients lost to follow-up, on the representativeness of the study populations and on-treatment compliance.

Of the ten articles included, six reported CVD outcomes [24-26, 28-30], two reported data on atherosclerosis as assessed by the surrogate marker cIMT [14, 31], and two reported outcomes related to endothelial function [23, 27]. Data were insufficient and outcomes were too varied to conduct a quantitative meta-analysis or to make systematic comparisons between ATV and other ARTs. Therefore, data were compiled qualitatively.

\section{Effect of ATV on CVD Outcomes}

Of the studies reporting CV outcomes, three were cohort studies and three were randomized-controlled trials (Table 1). Four of the studies included only treatment-naïve patients [24-26, 28], one included both treatment-experienced and -naïve patients [30], and one included only treatment-experienced patients [29]. The results of these studies are reported in Table 1.

The studies reporting the incidence of MI in HIV-infected patients showed that ATV was not 


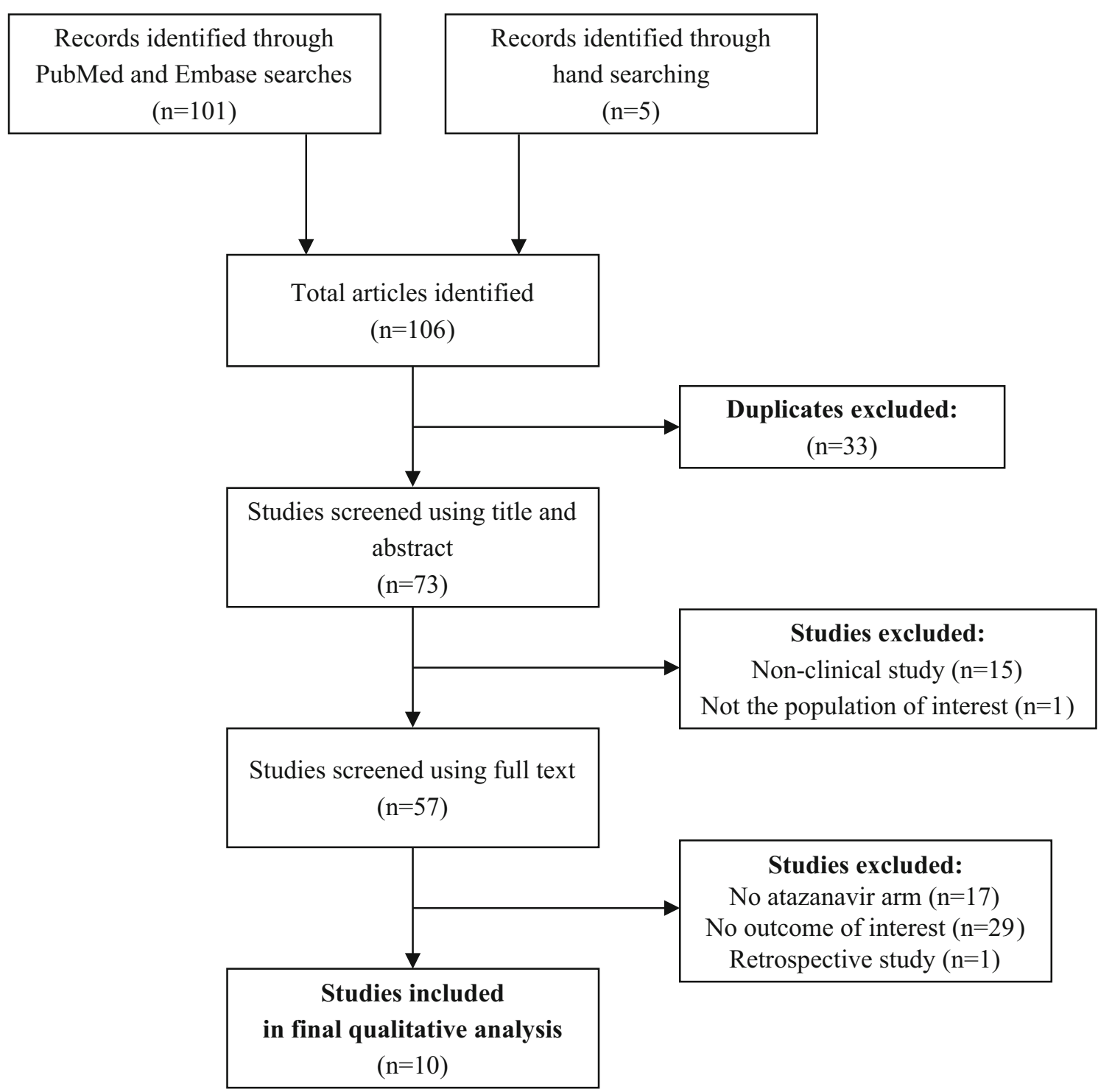

Fig. 1 PRISMA flow diagram of the study selection process

associated with an increased risk of acute MI. In a cohort of $>16,000$ treatment-naïve patients, patients initially treated with both boosted and unboosted ATV ( $n=543$; inverse probability weighted hazard ratio: $1.12 ; 95 \%$ CI $0.35-3.62)$ or LPV/r $(n=654 ; 0.92 ; 95 \%$ CI $0.26-3.22)$ did not have an increased rate of MI compared with the initial treatment with a non-nucleoside reverse-transcriptase inhibitor, whereas patients initially treated with $\mathrm{ABC}(n=611$; $2.05 ; 95 \%$ CI $0.72-5.86)$ trended towards an increased rate of MI compared with tenofovir disoproxil fumarate (TDF) [28]. Data from the treatment-naïve and -experienced patients included in the D:A:D (Data Collection on Adverse Events of Anti-HIV Drugs) study showed no relationship between ATV exposure (37,005 patient years) and an increased MI risk among HIV-infected patients compared with other AR agents, either overall (relative rate/ year 0.95; 95\% CI 0.86-1.04) or when ATV was given with or without ritonavir $(0.99 ; 95 \% \mathrm{CI}$ 


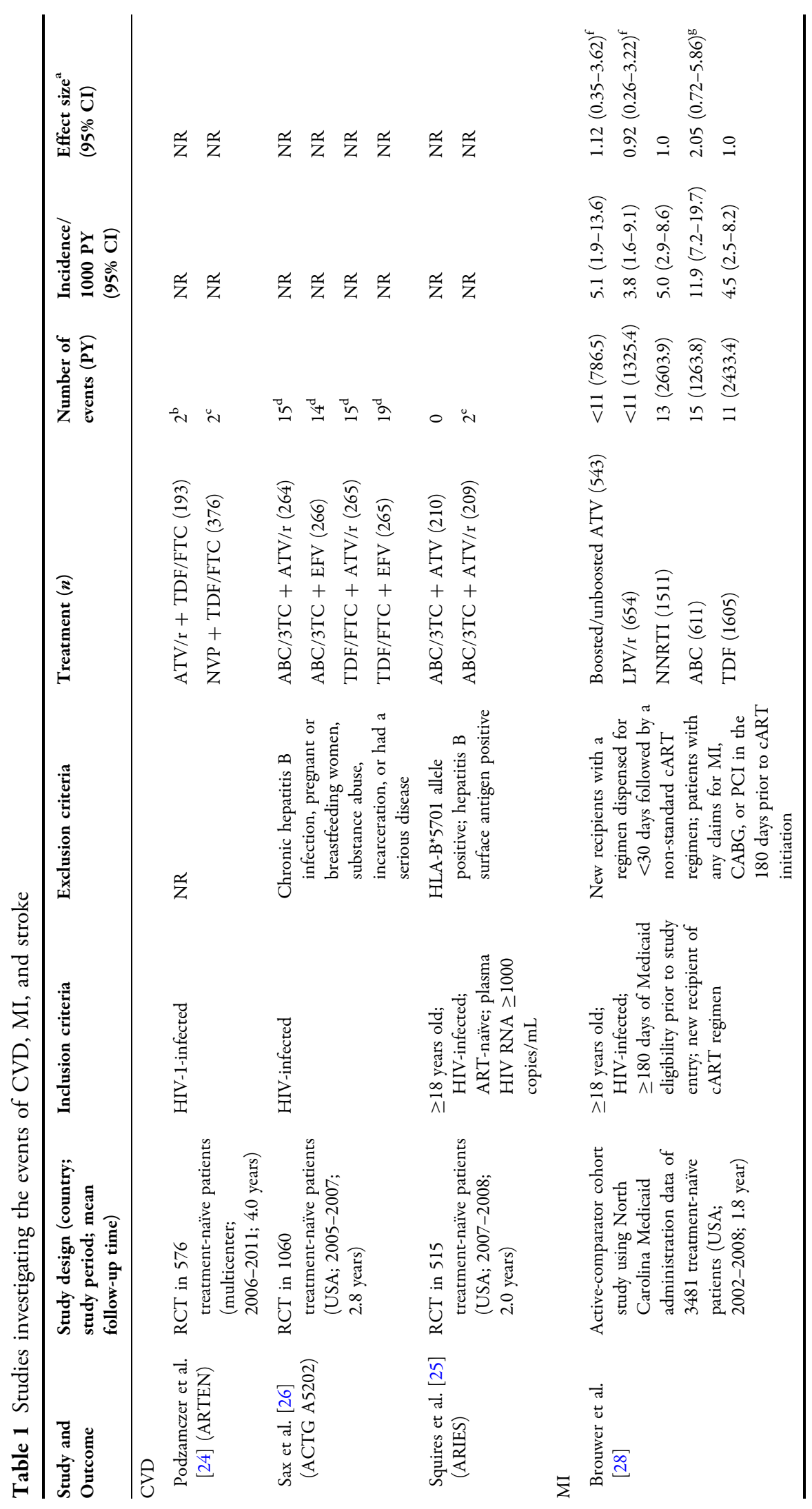




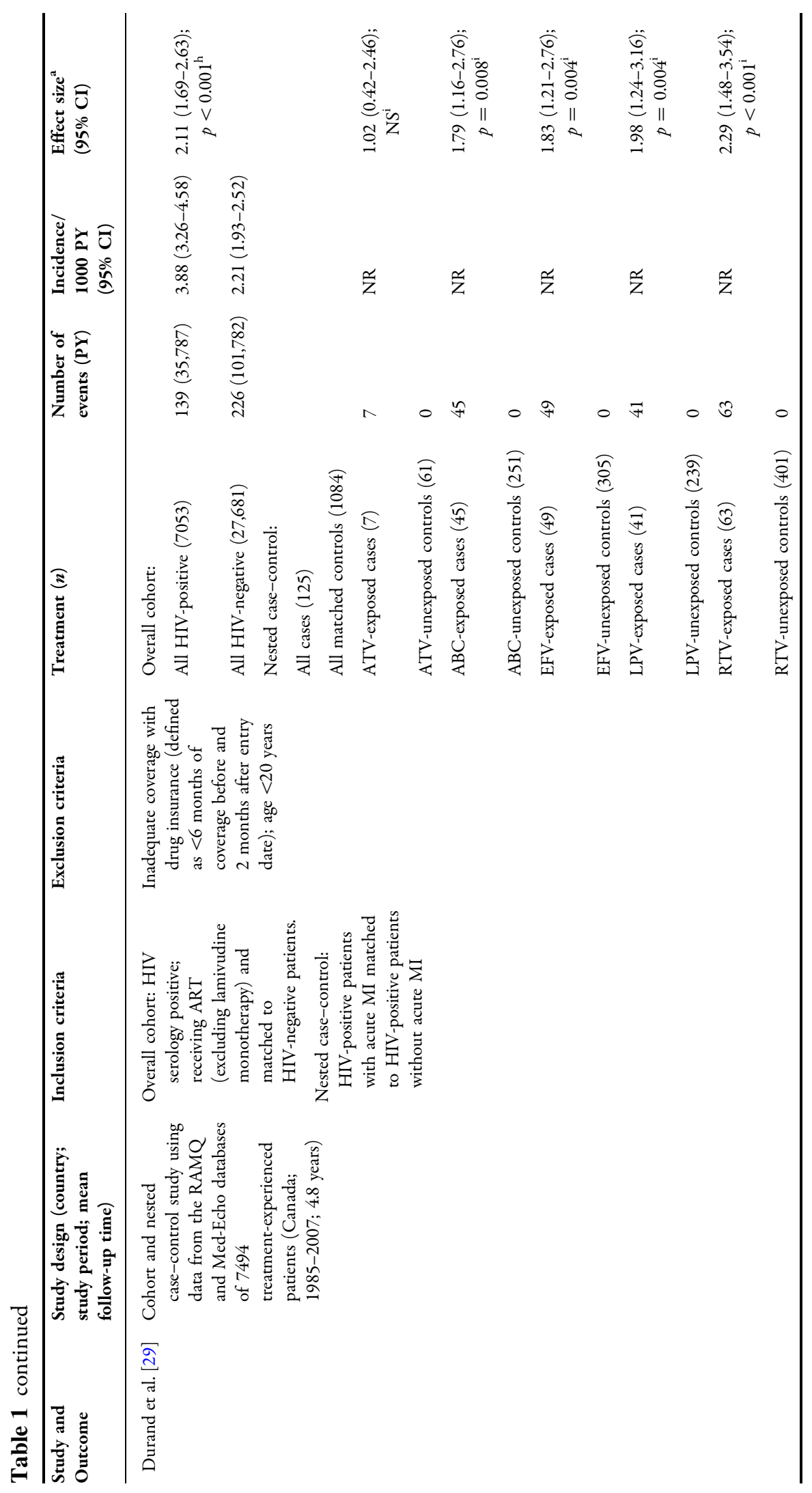




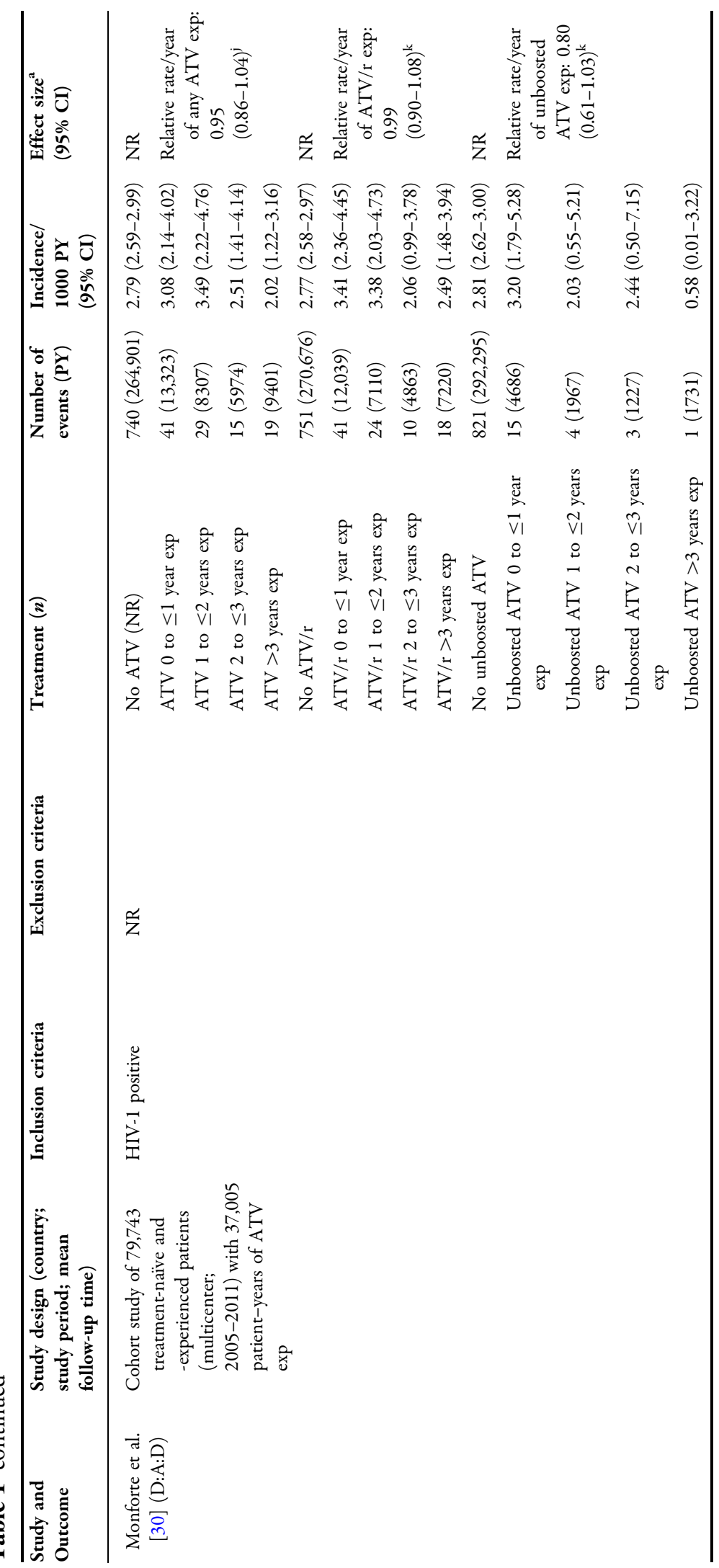




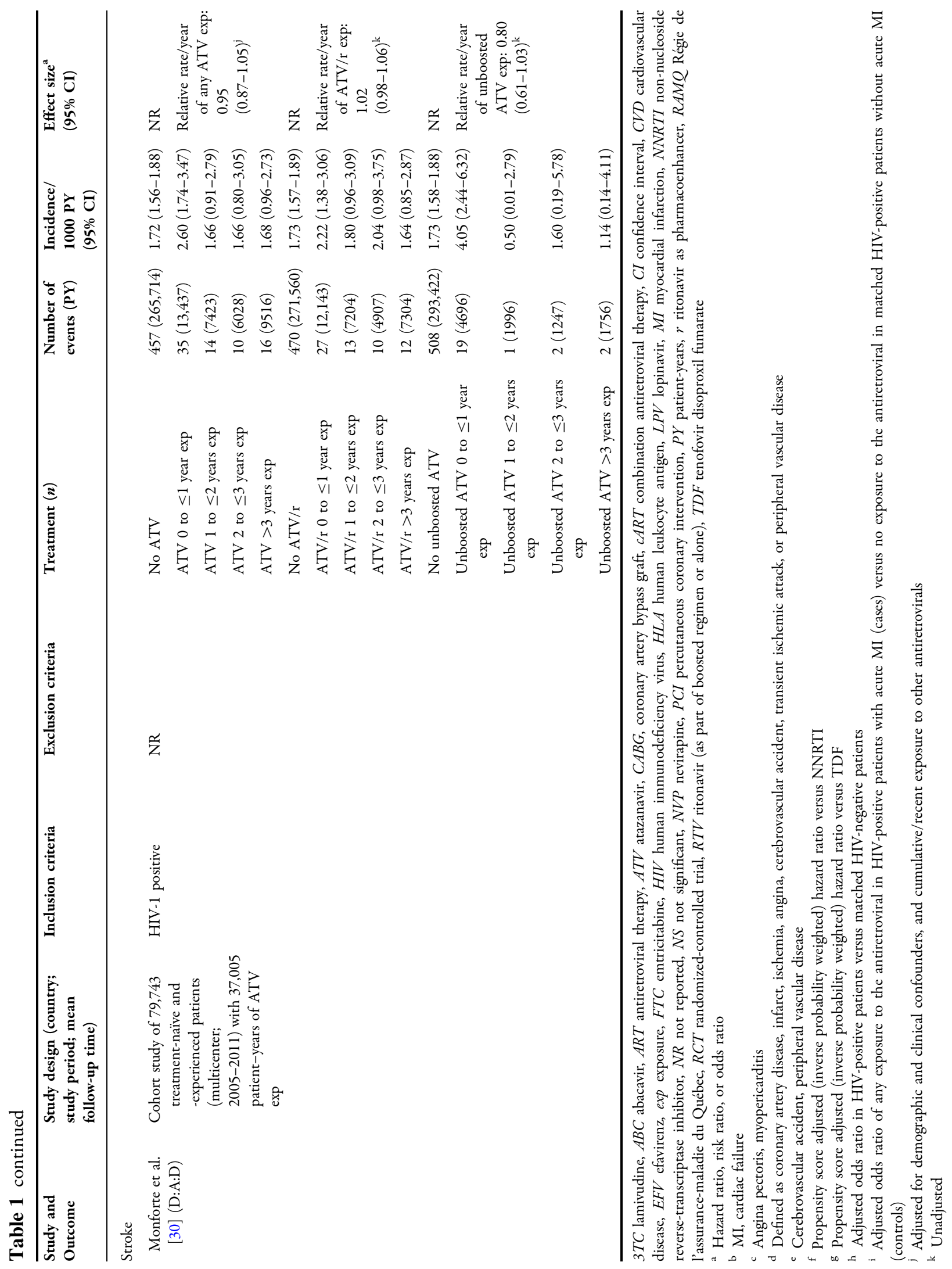




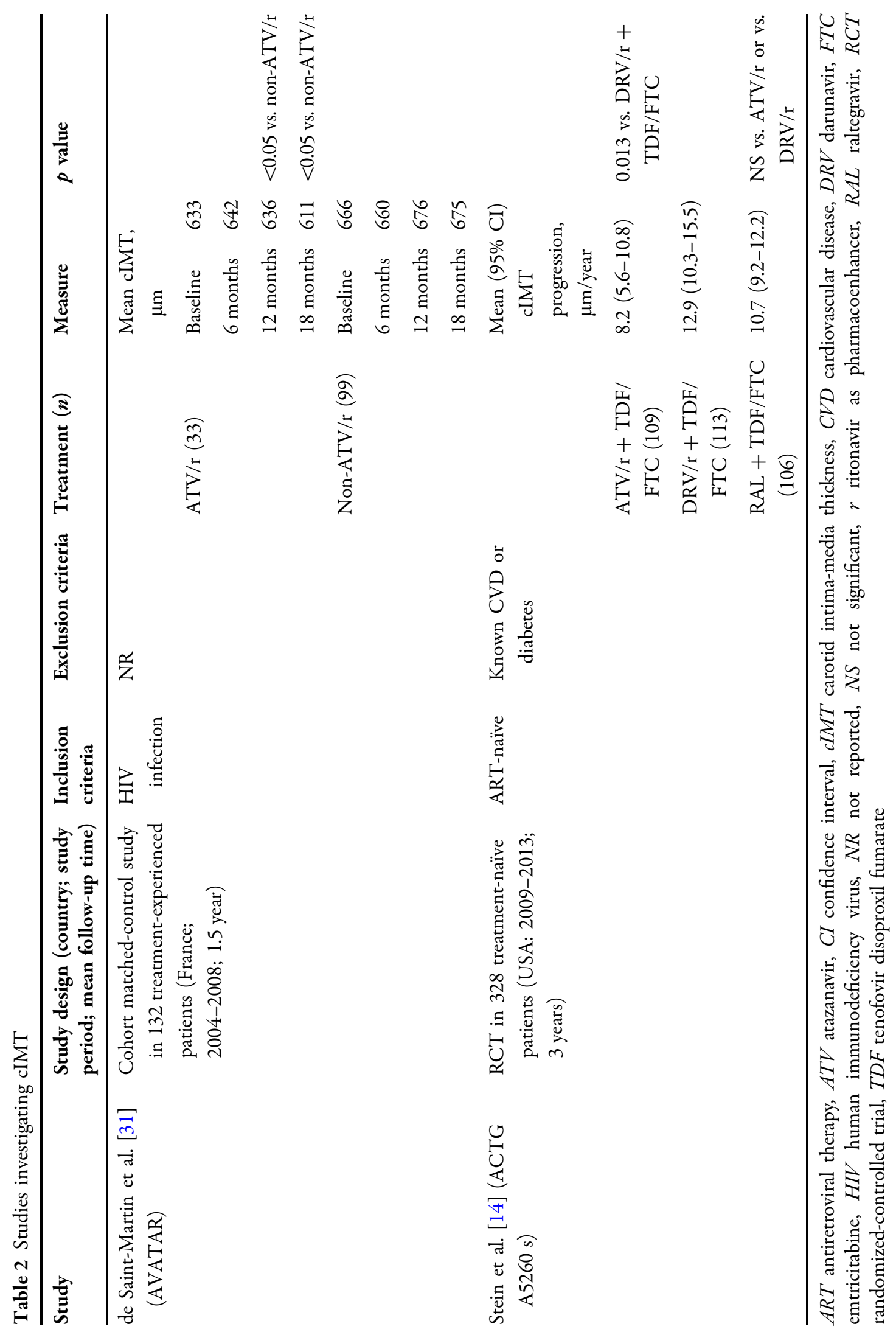




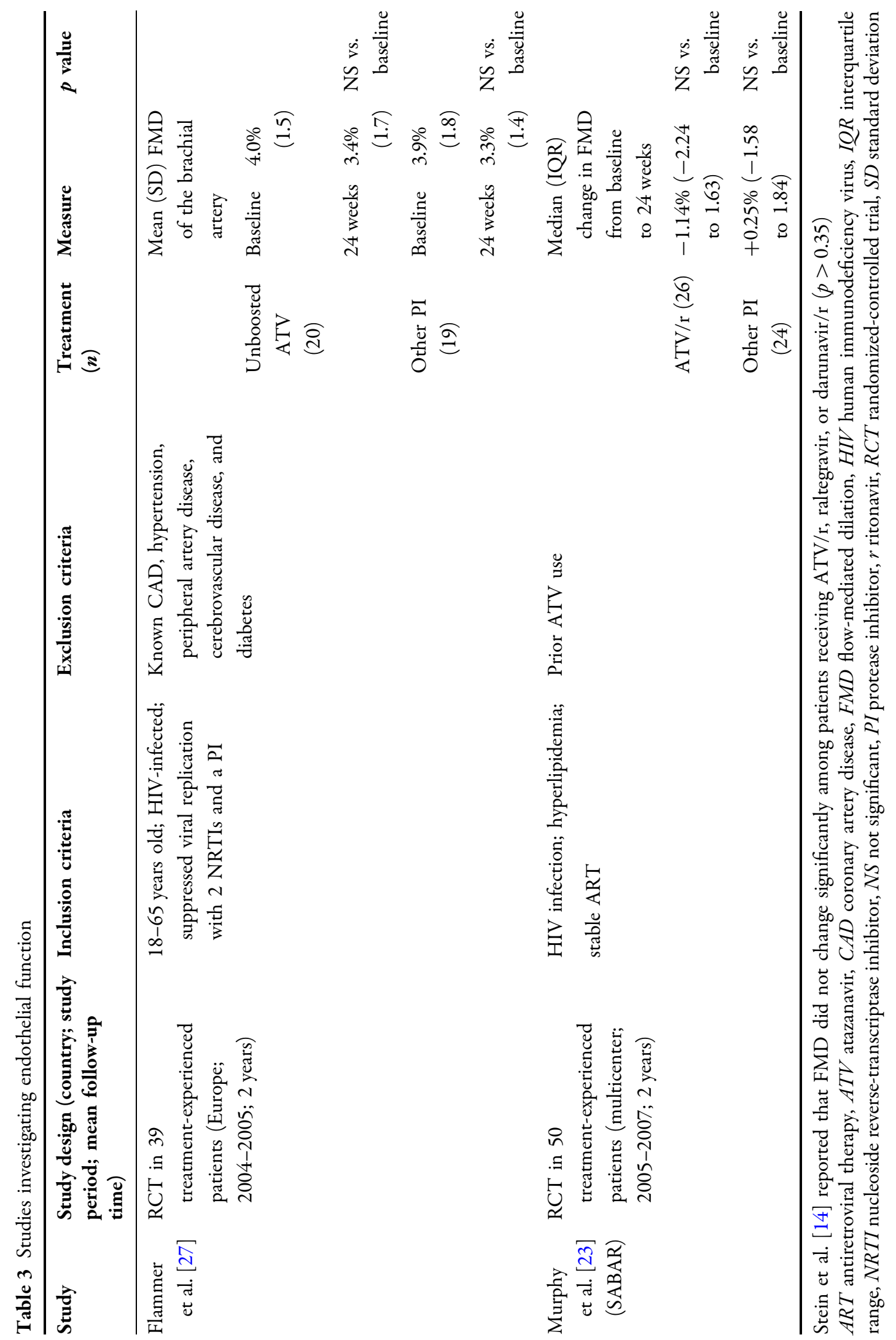


0.90-1.08 and $0.80 ; 95 \%$ CI 0.61-1.03, respectively) [30]. In the Durand et al. study, a cohort and nested control analysis of a public health insurance database included 7053 HIV-positive patients, and treatmentexperienced patients exposed to ATV with and without ritonavir (number exposed to ATV unknown) did not have an increased risk of MI compared with controls without ATV exposure $(p=0.96$; Table 1$)$ [29]. In this analysis, HIV-infected patients exposed to $\mathrm{ABC}, \mathrm{LPV}$, or ritonavir had an increased risk of acute MI (odds ratios 1.79, 1.98, and 2.29, respectively; $p<0.01$ vs. matched controls) [29].

Other CV endpoints were similarly unaffected by treatment with ATV. The D:A:D study examined the risk of stroke among HIV-infected patients exposed to ATV and found no association between ATV and an increased stroke risk (overall 0.95; 95\% CI 0.87-1.05; boosted 1.02; 95\% CI 0.98-1.06; unboosted $0.80 ; \quad 95 \%$ CI $0.61-1.03)$ [30] (Table 1).

Of the three randomized-controlled trials reporting a variety of $\mathrm{CV}$ endpoints (a composite of coronary artery disease, infarct, ischemia, angina, transient ischemic attack, cerebrovascular accident, or peripheral vascular disease [26] or unspecified CV adverse events $[24,25])$, there was no increase in $\mathrm{CV}$ events among HIV-infected patients receiving ATV $(n=1141)$. In the ACTG study A5202, there was a low incidence of $\mathrm{CV}$ events overall in patients receiving $\mathrm{ABC} /$ lamivudine or $\mathrm{TDF} /$ emtricitabine (FTC) plus ATV/r or efavirenz, with no difference between treatment groups [26]. In the studies reporting unspecified CV adverse events, there was one occurrence each of MI and cardiac failure among patients receiving ATV/r and one occurrence each of angina pectoris and myopericarditis among patients receiving nevirapine through 4 years of follow-up (Table 1) [24], and in the randomized open-label ARIES study (ClinicalTrials.gov identifier: NCT00440947), one occurrence of cerebrovascular accident and one of peripheral vascular disease in treatment-naïve patients treated with ATV with or without ritonavir in combination with ABC/lamivudine [25].

\section{Effect of ATV on Markers of Atherosclerosis}

Two studies investigated the effect of ATV on atherosclerosis using changes in cIMT. One was a prospective, randomized-controlled ACTG substudy (A5260 s) in treatment-naïve patients, while the other was a single-center, prospective matched-control cohort study in treatmentexperienced patients (Table 2) [14, 31].

Overall, the results of the cIMT measurements showed that treatment with ATV was associated with beneficial effects on cIMT progression compared with non-ATV-based regimens (Table 2). In the ACTG $5260 \mathrm{~s}$ study, patients received either TDF/FTC plus either ATV/r $(n=109), \mathrm{DRV} / \mathrm{r}$ $(n=113)$, or RAL $(n=106)$ for 144 weeks. At study end, increases in cIMT at the common carotid artery (primary intent-to-treat analysis) had progressed more slowly in patients receiving $\mathrm{ATV} / \mathrm{r}$ than in those receiving $\mathrm{DRV} / \mathrm{r}$ or RAL; however, differences reached statistical significance in the comparison with $\mathrm{DRV} / \mathrm{r}$ $(p=0.013)$ but not with RAL $(p=0.15)$ [14]. In the on-treatment analysis and at the carotid bifurcation, ATV/r was associated with significantly slower cIMT progression compared with both DRV and RAL [14]. In the cohort study, patients receiving regimens, including $\mathrm{ATV} / \mathrm{r} \quad(n=33)$, had significantly decreased cIMT compared with matched controls not receiving ATV/r $(n=99)$ after 12 
and 18 months of therapy ( $p \leq 0.05$ for both) [31]. The differences in cIMT progression remained significant after adjustment for differences in baseline demographics.

\section{Effect of ATV on Endothelial Function}

Two randomized-controlled trials in treatmentexperienced patients with HIV assessed endothelial function via flow-mediated dilation (FMD) of the brachial artery [23, 27] (Table 3). There was no impact of ATV therapy on endothelial function in these studies (Table 3). Both the randomized-controlled trials investigated switching from another PI to ATV (with or without ritonavir) with a trial duration of 24 weeks. In patients receiving unboosted ATV $(n=20)$, there was no change in FMD compared with the previous PI therapy $(p=0.40)$ [27], and patients receiving ATV/r $(n=26)$ did not demonstrate any significant change in FMD both from baseline and versus patients continuing on the previous $\mathrm{PI} / \mathrm{r}$ therapy $(p=0.64$ after adjustment for brachial artery diameter changes) [23]. Endothelial function was also measured in the ACTG A5260 s study. FMD did not change significantly among patients treated with ATV/r, RAL, or DRV/r. In addition, despite the differences seen in cIMT progression, there were no differences in FMD change between treatment arms [14].

\section{DISCUSSION}

This systematic literature review concerning ATV and CVD risk among patients with HIV showed no relationship between boosted and unboosted ATV and an increased risk for CV clinical outcome events, including $\mathrm{MI}$ and stroke. The use of ATV was associated with improvements in surrogate markers of atherosclerosis compared with non-ATV-based regimens, and the studies analyzed showed no impact of ATV on endothelial function.

This study did not investigate the impact of ATV on lipid levels in HIV-infected patients, as previous studies have explored this [11, 32]. In one systematic study, ATV was associated with lower lipid levels compared with a pooled cohort of other ritonavir-boosted PI regimens [11]. A second study of ritonavir-boosted PIs found that patients treated with ATV had cholesterol and triglyceride elevations that were lower than LPV or fosamprenavir but similar to saquinavir or DRV [32]. It is not entirely clear what contributions lipid elevations make to the overall CV risk. In the ACTG A5260 s study, ATV/r was associated with slower atherosclerosis progression compared to $\mathrm{DRV} / \mathrm{r}$ and RAL, despite RAL having smaller increases in lipid parameters than the boosted PI arms [14].

One potential explanation for this apparent discrepancy is ATV-induced hyperbilirubinemia, which is a common laboratory abnormality associated with ATV treatment. Bilirubin has been shown to have antioxidant, anti-inflammatory, and antiatherogenic properties and is thought to result in protection from CVD [33, 34]. Multiple studies have shown that individuals with Gilbert syndrome, a genetic deficiency in UGT1A1 resulting in chronic low level hyperbilirubinemia, have a reduced risk for $\mathrm{CV}$ events compared with the general population [35-38]. ATV has been shown to increase bilirubin levels and reduce levels of oxidative stress in HIV-infected patients compared with non-ATV-based treatment [23, 39]. Additional studies have also linked increasing bilirubin levels with slower atherosclerosis progression (as measured by cIMT) [14, 40], which suggests 
that there may be a relationship between ATV therapy, bilirubin levels and improvements in oxidative stress, atherosclerosis, and CV risk.

Due to its impact on bilirubin and the anti-atherosclerotic properties of bilirubin, ATV has been studied in non-HIV-infected patients with type 1 and 2 diabetes. In patients with type 1 diabetes, the initiation of ATV decreased lipid parameters and increased total plasma antioxidant capacity from baseline; however, similar to the studies of HIV-infected patients analyzed in this review, it did not result in an improvement in endothelial function [12]. In an experimental double-blind, placebo-controlled crossover study of HIV-negative patients with type 2 diabetes, ATV was associated with a significant improvement in both plasma antioxidant capacity and endothelial function [13].

Recent studies published after the search for this analysis was initiated are consistent with the results described here. In an analysis of data from HIV-infected patients in the Veterans Health Administration Clinical Case Registry, exposure to ATV was not associated with an increased risk of CV events [41]. Moreover, in a retrospective, longitudinal study of patients with HIV in Brazil, the recent boosted ATV exposure was associated with a decreased risk of CV outcome events [42]. Finally, in a retrospective analysis of the Hawaii Aging with HIV CV Study, patients receiving ATV/r had a slower progression of cIMT over 2 years compared with those not receiving ATV/r (9 $\mu \mathrm{m}$ vs. $22 \mu \mathrm{m} ; p<0.001$ ). These differences remained significant after adjusting for age, sex, and other CVD-related comorbidities $(p=0.012)$. Similar to the ACTG A5260 s study, there was a significant inverse correlation between increasing baseline total serum bilirubin and reduced cIMT progression [40].
This analysis has limitations. First, a small number of studies were identified with relatively few ATV patients. This combined with the variable forms of data presentation and outcomes prevented a meta-analysis from being performed. Second, data were insufficient to make any assessment of differences in $\mathrm{CV}$ outcomes with unboosted ATV versus ritonavir-boosted ATV. Third, studies solely investigating lipid levels were not included, and no attempt was made to account for concomitant medications, such as statins, or baseline comorbidities, or to weight evidence according to its source, i.e., from randomized-controlled studies or from studies performed under real-world conditions. Fourth, patients included in these analyses had variable durations of HIV infection, AR experience, and were exposed to a wide variety of ART regimens. Given the relatively limited information identified in this review, larger cohort studies are needed to better evaluate the relative risk of CVD with ATV versus other contemporary ARs in patients with HIV infection.

\section{CONCLUSIONS}

In this systematic review of CVD in HIV-infected patients receiving ATV, a limited number of studies were identified. In the identified studies, there was no increase in the risk or occurrence of adverse $\mathrm{CV}$ clinical outcome events with boosted and unboosted ATV compared to other AR agents. Endothelial function was not affected by ATV, while ATV use was associated with improvements in markers of atherosclerosis. These findings should be taken into consideration when selecting HIV treatment for individuals at increased risk of CVD. 


\section{ACKNOWLEDGMENTS}

Article processing charges for this study were funded by Bristol-Myers Squibb. All authors had full access to all of the data in this study and take complete responsibility for the integrity of the data and accuracy of the data interpretation. Ben Dale, Sheridan Henness, and Julian Martins of inscience Communications, Springer Healthcare (Philadelphia, PA, USA), provided medical writing support funded by Bristol-Myers Squibb (Princeton, NJ, USA).

All named authors meet the International Committee of Medical Journal Editors (ICMJE) criteria for authorship for this manuscript, take responsibility for the integrity of the work as a whole, and have given the final approval to the version to be published.

Disclosures. D Chow has no conflicts of interest to report and received no financial compensation for the writing of this article. C Shikuma received no financial compensation for the writing of this article but has received research support from Gilead Sciences, as well as investigational medications from Gilead Sciences, Merck, Tobira Therapeutics, and ViiV Healthcare. C Ritchings is an employee of and owns stock in Bristol-Myers Squibb. L Rosenblatt is an employee of and owns stock in Bristol-Myers Squibb. M Guo was an intern at Bristol-Myers Squibb at the time of this study.

Compliance with Ethics Guidelines. This article is based on previously conducted studies and does not involve any new studies of human or animal subjects performed by any of the authors.
Open Access. This article is distributed under the terms of the Creative Commons Attribution-NonCommercial 4.0 International License (http://creativecommons.org/licenses/ by-nc/4.0/), which permits any noncommercial use, distribution, and reproduction in any medium, provided you give appropriate credit to the original author(s) and the source, provide a link to the Creative Commons license, and indicate if changes were made.

\section{REFERENCES}

1. Antiretroviral Therapy Cohort Collaboration. Life expectancy of individuals on combination antiretroviral therapy in high-income countries: a collaborative analysis of 14 cohort studies. Lancet. 2008;372(9635):293-9.

2. Hanna DB, Ramaswamy C, Kaplan RC, et al. Trends in cardiovascular disease mortality among persons with HIV in New York City, 2001-2012. Clin Infect Dis. 2016. doi:10.1093/cid/ciw470.

3. Currier JS, Lundgren JD, Carr A, et al. Epidemiological evidence for cardiovascular disease in HIV-infected patients and relationship to highly active antiretroviral therapy. Circulation. 2008;118(2):e29-35.

4. Rotger M, Glass TR, Junier T, et al. Contribution of genetic background, traditional risk factors, and HIV-related factors to coronary artery disease events in HIV-positive persons. Clin Infect Dis. 2013;57(1):112-21.

5. Duprez DA, Neuhaus J, Kuller LH, et al. Inflammation, coagulation and cardiovascular disease in HIV-infected individuals. PLoS One. 2012;7(9):e44454.

6. Grinspoon S, Carr A. Cardiovascular risk and body-fat abnormalities in HIV-infected adults. N Engl J Med. 2005;352(1):48-62.

7. Phillips AN, Carr A, Neuhaus J, et al. Interruption of antiretroviral therapy and risk of cardiovascular disease in persons with HIV-1 infection: exploratory analyses from the SMART trial. Antivir Ther. 2008;13(2):177-87. 
8. Bavinger C, Bendavid E, Niehaus K, et al. Risk of cardiovascular disease from antiretroviral therapy for HIV: a systematic review. PLoS One. 2013;8(3):e59551.

9. Friis-Moller N, Reiss P, Sabin CA, et al. Class of antiretroviral drugs and the risk of myocardial infarction. N Engl J Med. 2007;356(17):1723-35.

10. Worm SW, Sabin C, Weber R, et al. Risk of myocardial infarction in patients with HIV infection exposed to specific individual antiretroviral drugs from the 3 major drug classes: the data collection on adverse events of anti-HIV drugs (D:A:D) study. J Infect Dis. 2010;201(3):318-30.

11. Carey D, Amin J, Boyd M, Petoumenos K, Emery S. Lipid profiles in HIV-infected adults receiving atazanavir and atazanavir/ritonavir: systematic review and meta-analysis of randomized controlled trials. J Antimicrob Chemother. 2010;65(9):1878-88.

12. Milian J, Goldfine AB, Zuflacht JP, Parmer C, Beckman JA. Atazanavir improves cardiometabolic measures but not vascular function in patients with long-standing type 1 diabetes mellitus. Acta Diabetol. 2015;52(4):709-15.

13. Dekker D, Dorresteijn MJ, Pijnenburg M, et al. The bilirubin-increasing drug atazanavir improves endothelial function in patients with type 2 diabetes mellitus. Arterioscler Thromb Vasc Biol. 2011;31(2):458-63.

14. Stein JH, Ribaudo HJ, Hodis $\mathrm{HN}$, et al. A prospective, randomized clinical trial of antiretroviral therapies on carotid wall thickness. AIDS. 2015;29(14):1775-83.

15. Mayer M. Association of serum bilirubin concentration with risk of coronary artery disease. Clin Chem. 2000;46(11):1723-7.

16. Lennox JL, Landovitz RJ, Ribaudo HJ, et al. Efficacy and tolerability of 3 nonnucleoside reverse transcriptase inhibitor-sparing antiretroviral regimens for treatment-naive volunteers infected with HIV-1: a randomized, controlled equivalence trial. Ann Intern Med. 2014;161(7):461-71.

17. O'Leary DH, Polak JF, Kronmal RA, Manolio TA, Burke GL, Wolfson Jr SK. Carotid-artery intima and media thickness as a risk factor for myocardial infarction and stroke in older adults. Cardiovascular Health Study Collaborative Research Group. N Engl J Med. 1999;340(1):14-22.

18. Goldberger ZD, Valle JA, Dandekar VK, Chan PS, Ko DT, Nallamothu BK. Are changes in carotid intima-media thickness related to risk of nonfatal myocardial infarction? A critical review and meta-regression analysis. Am Heart J. 2010;160(4):701-14.

19. Shimbo D, Grahame-Clarke C, Miyake Y, et al. The association between endothelial dysfunction and cardiovascular outcomes in a population-based multi-ethnic cohort. Atherosclerosis. 2007;192(1): 197-203.

20. Hadi HA, Carr CS, Al Suwaidi J. Endothelial dysfunction: cardiovascular risk factors, therapy, and outcome. Vasc Health Risk Manag. 2005;1(3):183-98.

21. National Institutes of Clinical Excellence. The guidelines manual 2012. Appendix C: Methodology checklist: randomised controlled trials. Available from https://www.nice.org.uk/ process/pmg6/resources/the-guidelines-manualappendices-bi-1967364/chapter/2-appendix-c-method ology-checklist-randomised-controlled-trials. Accessed 18 August 2016.

22. Downs $\mathrm{SH}$, Black N. The feasibility of creating a checklist for the assessment of the methodological quality both of randomised and non-randomised studies of health care interventions. J Epidemiol Commun Health. 1998;52(6):377-84.

23. Murphy RL, Berzins B, Zala C, et al. Change to atazanavir/ritonavir treatment improves lipids but not endothelial function in patients on stable antiretroviral therapy. AIDS. 2010;24(6): 885-90.

24. Podzamczer D, Andrade-Villanueva J, Clotet B, et al. Lipid profiles for nevirapine vs. atazanavir/ ritonavir, both combined with tenofovir disoproxil fumarate and emtricitabine over 48 weeks, in treatment-naive HIV-1-infected patients (the ARTEN study). HIV Med. 2011;12(6):374-82.

25. Squires KE, Young B, Dejesus E, et al. Similar efficacy and tolerability of atazanavir compared with atazanavir/ritonavir, each with abacavir/ lamivudine after initial suppression with abacavir/ lamivudine plus ritonavir-boosted atazanavir in HIV-infected patients. AIDS. 2010;24(13): 2019-27.

26. Sax PE, Tierney C, Collier AC, et al. Abacavir/ lamivudine versus tenofovir DF/emtricitabine as part of combination regimens for initial treatment of HIV: final results. J Infect Dis. 2011;204(8): 1191-201.

27. Flammer AJ, Vo NT, Ledergerber B, et al. Effect of atazanavir versus other protease inhibitor-containing antiretroviral therapy on endothelial function in HIV-infected persons: randomised controlled trial. Heart. 2009;95(5):385-90. 
28. Brouwer ES, Napravnik S, Eron Jr JJ, et al. Effects of combination antiretroviral therapies on the risk of myocardial infarction among HIV patients. Epidemiology. 2014;25(3):406-17.

29. Durand M, Sheehy O, Baril JG, Lelorier J, Tremblay CL. Association between HIV infection, antiretroviral therapy, and risk of acute myocardial infarction: a cohort and nested case-control study using Quebec's public health insurance database. J Acquir Immun Defic Syndr. 2011;57(3):245-53.

30. Monforte A, Reiss P, Ryom L, et al. Atazanavir is not associated with an increased risk of cardio or cerebrovascular disease events. AIDS. 2013;27(3): 407-15.

31. de Saint-Martin L, Bressollette L, Perfezou P, et al. Impact of atazanavir-based HAART regimen on the carotid intima-media thickness of HIV-infected persons: a comparative prospective cohort. AIDS. 2010;24(18):2797-801.

32. Hill A, Sawyer W, Gazzard B. Effects of first-line use of nucleoside analogues, efavirenz, and ritonavir-boosted protease inhibitors on lipid levels. HIV Clin Trials. 2009;10(1):1-12.

33. Tatami YSS, Ishii H, Shibata Y, Osugi N, Ota T, et al. Impact of serum bilirubin levels on carotid atherosclerosis in patients with coronary artery disease. IJC Metab Endocr. 2015;5:24-7.

34. Kang SJ, Lee C, Kruzliak P. Effects of serum bilirubin on atherosclerotic processes. Ann Med. 2014;46(3): 138-47.

35. Vitek L, Jirsa M, Brodanova $M$, et al. Gilbert syndrome and ischemic heart disease: a protective effect of elevated bilirubin levels. Atherosclerosis. 2002;160(2):449-56.
36. Horsfall LJ, Nazareth I, Pereira SP, Petersen I. Gilbert's syndrome and the risk of death: A population-based cohort study. J Gastroenterol Hepatol. 2013;28(10):1643-7.

37. Perlstein TS, Pande RL, Creager MA, Weuve J, Beckman JA. Serum total bilirubin level, prevalent stroke, and stroke outcomes: NHANES 1999-2004. Am J Med. 2008;121(9):781-8 e781.

38. Lin JP, O'Donnell CJ, Schwaiger JP, et al. Association between the UGT1A1*28 allele, bilirubin levels, and coronary heart disease in the Framingham Heart Study. Circulation. 2006;114(14): 1476-81.

39. Estrada V, Monge S, Gomez-Garre D, et al. Comparison of oxidative stress markers in HIV-infected patients on efavirenz or atazanavir/ ritonavir-based therapy. J Int AIDS Soc. 2014;17(4 Suppl 3):19544.

40. Chow D, Kohorn L, Souza S, et al. Atazanavir use and carotid intima media thickness progression in HIV: potential influence of bilirubin. AIDS. 2016;30(4):672-4.

41. Desai M, Joyce V, Bendavid E, et al. Risk of cardiovascular events associated with current exposure to HIV antiretroviral therapies in a US veteran population. Clin Infect Dis. 2015;61(3): $445-52$.

42. Diaz CM, Segura ER, Luz PM, et al. Traditional and HIV-specific risk factors for cardiovascular morbidity and mortality among HIV-infected adults in Brazil: a retrospective cohort study. BMC Infect Dis. 2016;16:376. 